\title{
ANALYSIS OF THE ESR SPECTRUM OF MANGANESE (II) IN THE LAYERED COMPOUND $\mathrm{Cd}_{2} \mathrm{P}_{2} \mathrm{Se}_{6}$
}

\author{
D.A. CLEARY, A.H. FRANCIS \\ Department of Chemistry, University of Michigan, Ann Arbor, MI 48109, USA
}

and

\section{E. LIFSHITZ}

Weizmann Institute of Science, Rehovot, Israel

Received 4 November 1985

\begin{abstract}
The Q-band and X-band ESR spectra of $\mathrm{Mn}^{2+}$ in the layered compound $\mathrm{Cd}_{2} \mathrm{P}_{2} \mathrm{Se}_{6}$ are reported. The $\mathrm{Mn}^{2+}$ is in a trigonal environment coordinated to six selenium atoms. The parameters of the spin hamiltonian have been determined and reasonable agreement is obtained between theory and experiment with $g_{\|}=2.01, g_{\perp}=2.05,|D|=0.286 \mathrm{~cm}^{-1},|A|=70.0 \times 10^{-4}$ $\mathrm{cm}^{-1}$. The results of the ESR analysis are compared with previous studies of the analogous layered compound $C \mathrm{~d}_{2} P_{2} S_{\gamma}$. The $\mathrm{Mn}$ (II) crystal-field splitting parameter $D$ increases approximately eight-fold from that in the sulfide lattice. The exceptionally large crystal-field splitting is correlated with the layered structure of the lattice and the $\mathrm{Mn}-\mathrm{Se}$ bond covalency. It is concluded that the dominant effect is that of the bond covalency.
\end{abstract}

\section{Introduction}

Because of its extreme sensitivity to the local environment, the zero-ficld splitting of ${ }^{6} \mathrm{~S}_{5 / 2}$ ions such as $\mathrm{Fe}^{3+}$ and $\mathrm{Mn}^{2+}$ has received considerable theoretical and experimental attention. Sharma et al. [1] published a series of papers describing the effects of several factors that influence the zerofield splitting terms $D$ and $E$ [see discussion and eq. (1) below]. These include the covalency of the metal-ligand bond and the admixture of metal excited states with the ${ }^{6} S_{5 / 2}$ ground state. Nicholson and Burns [2] compared the axial crystal field obtained from Mössbauer absorption experiments with the $D$ values from ESR studies and found "serious disagreement" between experiment and theory for the dependence of $D$ upon the axial component of the crystal field. Schlaak and Weiss [3] determined $D$ for $\mathrm{Mn}^{2+}$ doped in $\mathrm{CdGa}_{2} \mathrm{~S}_{4}$ and $\mathrm{CdGa}_{2} \mathrm{Se}_{4}$ from ESR measurements and concluded that the more covalent $\mathrm{Mn}-\mathrm{Se}$ bond was responsible for the much larger value of $D$ in the selenide.

Large $\mathrm{Mn}^{2+}$ crystal-field splittings have been observed in materials where either the metalligand bonding was highly covalent or exceptionally high values of the axial crystal field were present. One of the largest value of $|D|$ reported for $\mathrm{Mn}^{2+}$ appears to be $|D|=0.544 \mathrm{~cm}^{-1}$ for a $\mathrm{Mn}^{2+}-\mathrm{V}_{\mathrm{O}}$ center in $\mathrm{SrTiO}_{3}$ [4]. The $\mathrm{Mn}^{2+}$ is adjacent to an $\mathrm{O}^{2-}$ vacancy and there is little doubt, in this example, that the unusually strong axial field, rather than bond covalency, is responsible for the exceptionally high value of $D$. The largest $|D|$ value reported to date for $\mathrm{Mn}$ (II) $\left(0.86 \mathrm{~cm}^{-1}\right)$ was measured for substitutional impurity ions in $\mathrm{MgTe}_{2}$ [5], where covalent bonding contributions are expected to be substantial.

In this paper we report the analysis of the ESR spectrum of $\mathrm{Mn}^{2+}$ in $\mathrm{Cd}_{2} \mathrm{P}_{2} \mathrm{Se}_{6}$. These results, combined with our earlier measurements for $\mathrm{Mn}^{2+}$ in $\mathrm{Cd}_{2} \mathrm{P}_{2} \mathrm{~S}_{6}$, give a clear example of the influence 
of covalency upon the zero-field splitting parameter, $D$.

\section{Experimental}

Single crystals of $\mathrm{Cd}_{2} \mathrm{P}_{2} \mathrm{Se}_{6}$ containing $1 \mathrm{~mol} \%$ Mn(II) were grown from the elements. A total charge of 1.000 gram was prepared to which 0.01 gram of iodine was added. The sample was evacuated and sealed off in a Vycor tube $(10 \times 1.5$ $\mathrm{cm}^{2}$ ). The synthesis was carried out in a single-zone furnace at $700^{\circ} \mathrm{C}$ for three days.

$\mathrm{X}$-band ESR spectra were recorded with a Bruker ER 200E-SRC spectrometer equipped with a $\mathrm{TM}_{110}$ cavity. Q-band spectra were recorded on a Varian 4503 spectrometer using a $T_{011} c y-$ lindrical cavity.

ESR spectra were recorded using single crystals of approximate dimensions $2 \times 2 \times 0.1 \mathrm{~mm}^{3}$ containing approximately $1 \mathrm{~mol} \% \mathrm{Mn}(\mathrm{II})$. Excitation frequencies of 9.7 and $35.25 \mathrm{GHz}$ were employed. The crystals were oriented within the microwave cavity with the axis normal to the basal plane (crystallographic $c$ axis) either parallel or perpendicular to the magnetic field. Low-temperature spectra were recorded using a gas-flow liquidnitrogen heat exchanger.

\section{Results}

The ESR spectrum of $\mathrm{Cd}_{2} \mathrm{P}_{2} \mathrm{Se}_{8}(1 \mathrm{~mol} \% \mathrm{Mn})$ obtained at $\mathrm{X}$-band in the perpendicular orientation is shown in fig. 1a. The spectrum consists of two hyperfine sextets centered at 1178 and 3650 $\mathrm{G}$, plus a very weak sextet centered at $9715 \mathrm{G}$. The spectrum for the parallel orientation, shown in fig. $1 b$, consists of two hyperfine multiplets centered at 2541 and $3409 \mathrm{G}$.

The Q-band spectra are shown in figs. 2a and 2b. The perpendicular spectrum exhibits all 30 of the expected hyperfine resonance lines of a highspin, $d^{5}, M n(I I)$. The parallel spectrum shows only three of the five sextets expected. The perpendicular Q-band spectrum is invariant to rotation about the $c$ axis, consistent with the crystal structure of the host lattice, which is trigonal with the three-

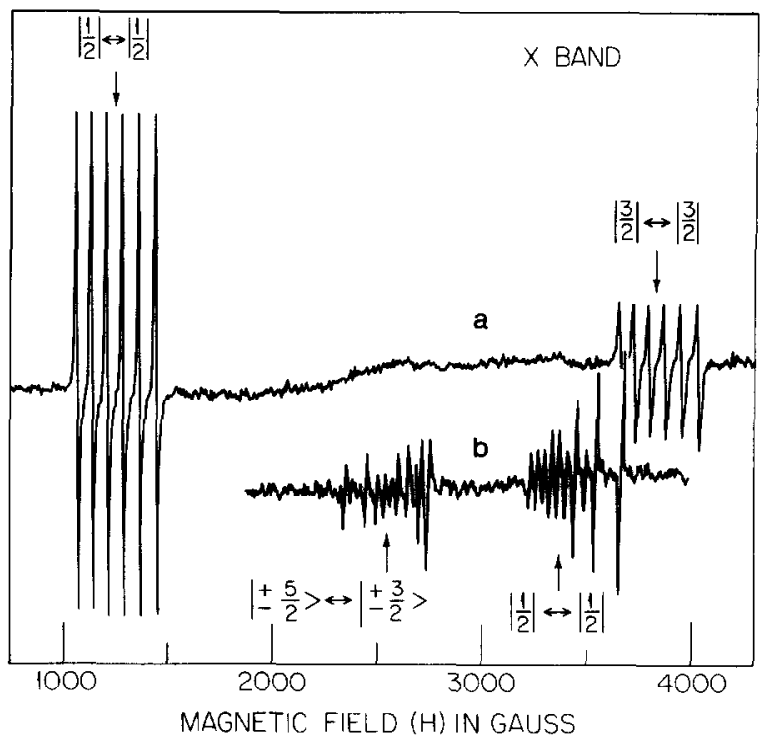

Fig. 1. The X-band ESR spectrum of $\mathrm{Cd}_{2} \mathrm{P}_{2} \mathrm{Se}_{6}$ obtained with: (a) $H$ perpendicular to $c$ and (b) $H$ parallel to $c$.

fold axis parallel to the $c$ axis. Also, the perpendicular spectrum is relatively insensitive to temperature down to $133 \mathrm{~K}$.

The ESR spectrum of $\mathrm{Mn}$ (II) centers in the isomorphous sulfide lattice $\mathrm{Cd}_{2} \mathrm{P}_{2} \mathrm{~S}_{6}$ obtained with $H$ perpendicular to $z$ is shown in fig. 3 .

\section{Discussion}

\subsection{Spin-hamiltonian analysis}

The ESR spectra may be interpreted with the aid of the following hamiltonian for $\mathrm{Mn}(\mathrm{II}), \mathrm{d}^{5}(S$ $=5 / 2$ ) in an axially symmetric crystal field [6].

$$
\begin{aligned}
\hat{H}= & \boldsymbol{H} \cdot \boldsymbol{g} \cdot \boldsymbol{S}+D\left[S_{z}^{2}-35 / 12\right] \\
& +(a / 6)\left[S_{z}^{4}+S_{y}^{4}+S_{x}^{4}-707 / 16\right] \\
& -(7 F / 36)\left[(81 / 14) S_{z}^{4}+81 / 16\right]+\boldsymbol{S} \cdot \mathbf{A} \cdot \boldsymbol{I} .
\end{aligned}
$$

$x, y$ and $z$ refer to the crystallographic $\{100\}$, $\{010\}$ and $\{001\}$ directions; $a, D$ and $F$ are the cubic, second- and fourth-order axial crystal field parameters, respectively. 


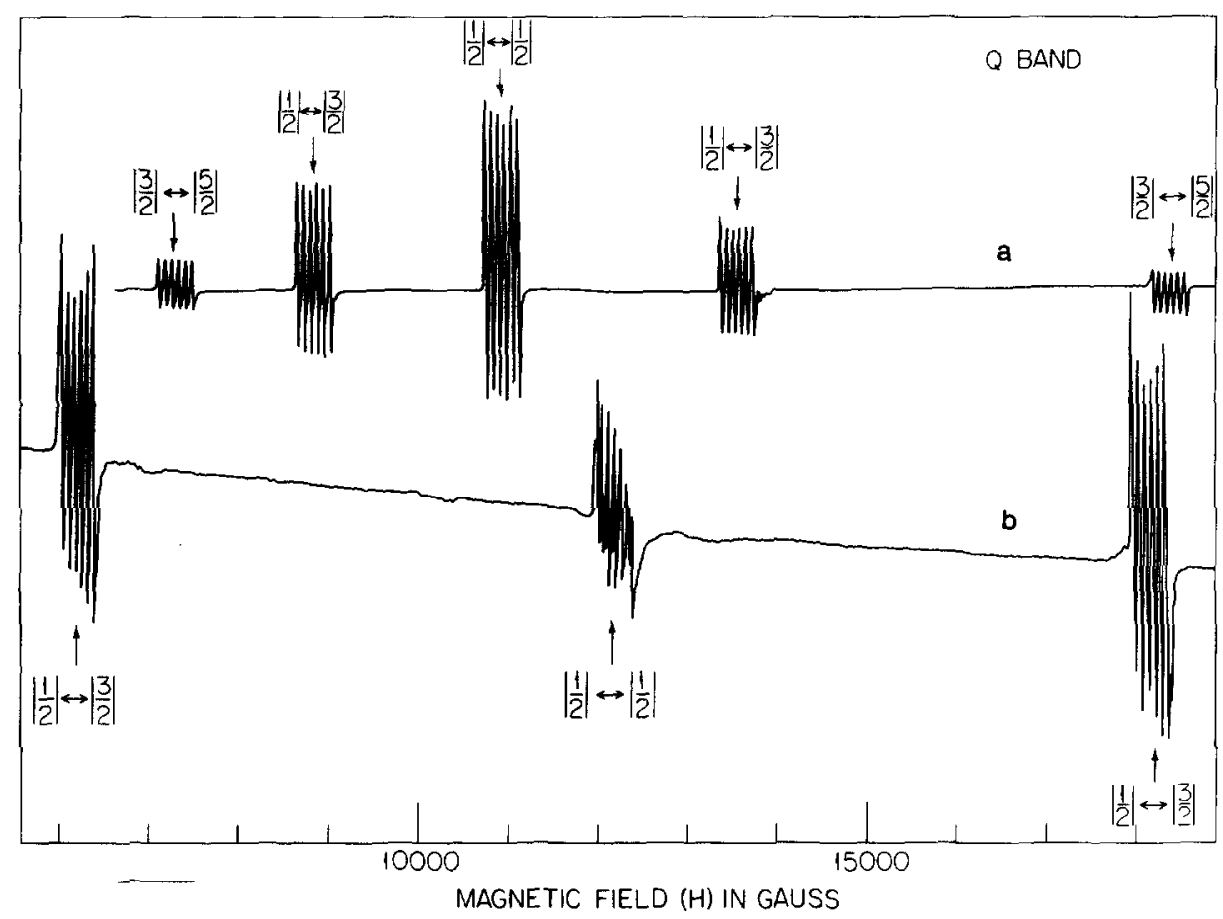

Fig. 2. The Q-band ESR spectrum of $\mathrm{Cd}_{2} \mathrm{P}_{2} \mathrm{Se}_{6}$ obtained with: (a) $H$ perpendicular to $c$ and (b) $H$ parallel to $c$. The arrows indicate the positions of the calculated fine-structure resonances.

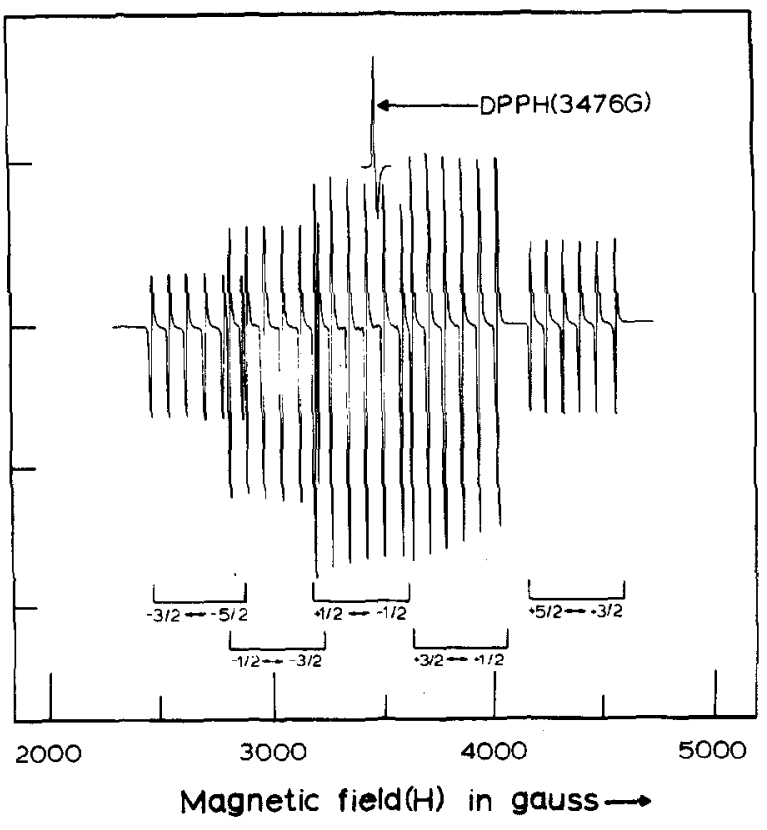

Fig. 3. The X-band ESR spectrum of $\mathrm{Cd}_{2} \mathrm{P}_{2} \mathrm{~S}_{6}$ with $H$ perpendicular to $c$.
Analysis of the X-band ESR spectra (fig. 1) using the hamiltonian (1) proved difficult due to the complexity of the spectrum obtained with $H$ parallel to $z$ and the limited fine-structure resonances in the spectrum with $H$ perpendicular to $z$. The low-field sextet in the $H$ perpendicular to $z$ spectrum arises from transitions between components of the $+1 / 2$ and $-1 / 2$ Kramers doublet. As expected, the powder spectrum at $273 \mathrm{~K}$ shows only these six lines. The mid-field transitions are between the levels of the $+3 / 2$ and $-3 / 2$ Kramers doublet, which are forbidden in the parallel spectrum, but are allowed in the perpendicular spectrum due to mixing of states [4]. The weak high-field transitions are between the $+5 / 2$ and $-5 / 2$ levels.

The $H$ parallel to $z$ spectrum consists of the $\pm 1 / 2 \leftrightarrow \pm 3 / 2$ and $\mp 1 / 2 \leftrightarrow \pm 1 / 2$ fine structure transitions. The sign ambiguity results from uncertainty in the sign of $D$. A positive value of $D$ is adopted for the remaining discussion and is consistent with the $-1 / 2 \leftrightarrow-3 / 2$ assignment. The 
complexity of the spectrum is due to the crossing of $+1 / 2$ and $-3 / 2$ spin states. The perturbation is severe and results in highly irregular hyperfine intervals.

The energy level congestion is largely removed in the higher fields employed with the Q-band spectrometer. For the Zeeman energy large compared to $a, D$ and $F$, eq. (1) yields the following expressions for the fine-structure spacing when $H$ is parallel to $z[13]$.

$$
\begin{aligned}
& \Delta H_{1}=[2 D-3(a-F)] / g_{11} \beta, \\
& \Delta H_{2}=[2 D-1.67(a-F)] / g_{11} \beta, \\
& \Delta H_{3}=[2 D+1.67(a-F)] / g_{11} \beta, \\
& \Delta H_{4}=[2 D+3(a-F)] / g_{11} \beta .
\end{aligned}
$$

Unfortunately, only three of the five hyperfine multiplets could be observed experimentally in this orientation due to the limited magnetic field strength available. If a larger field had been available, a fourth sextet at $\approx 24 \mathrm{kG}$ would be observed and the spacing between it and the one at $18 \mathrm{kG}$ would allow a value of $(a-F)$ to be calculated. It was possible, however, to obtain an initial estimate of $|D|$ with the assumption that $|D| \gg(a-F)$.

The Q-band spectrum obtained with $H$ perpendicular to $z$ may be described approximately using relations derived by Meirovitch and Poupko [7] from a second-order perturbation treatment. The field intervals between the five hyperfine multiplets are:

$$
\begin{aligned}
& \Delta H_{1}=\left(D-2.25 D^{2} / \hbar \omega\right) / g_{\perp} \beta, \\
& \Delta H_{2}=\left(D-0.75 D^{2} / \hbar \omega\right) / g_{\perp} \beta, \\
& \Delta H_{3}=\left(D+0.75 D^{2} / \hbar \omega\right) / g_{\perp} \beta, \\
& \Delta H_{4}=\left(D+2.25 D^{2} / \hbar \omega\right) / g_{\perp} \beta .
\end{aligned}
$$

The quadratic dependence of the interval between fine-structure multiplets is clearly evident in fig. $2 a$ and is accurately reproduced by eqs. (3) with $|D|=0.286 \mathrm{~cm}^{-1}$.

The asymmetry in the fine-structure interval arises from the $D^{2} / h \omega$ term which is equal to $0.0696 \mathrm{~cm}^{-1}$ for $|D|=0.286 \mathrm{~cm}^{-1}$ at Q-band and results in a pronounced variation in the fine- structure interval evident in fig. $2 b$. The same effect is observed in the ESR spectrum of $\mathrm{Mn}(\mathrm{II})$ in $\mathrm{Cd}_{2} \mathrm{P}_{2} \mathrm{~S}_{6}[8-10]$ recorded at $\mathrm{X}$-band (fig. 3). The smaller $|D|$ value of $0.0365 \mathrm{~cm}^{-1}$ yields $D^{2} / \hbar \omega=0.0041 \mathrm{~cm}^{-1}$ and the corresponding smaller variation in the interval spacing seen in fig. 3.

With the assumptions that $|D| \gg a$ and $D \gg$ $F$, the final values of $g$ and $|D|$ were determined by repeated diagonalization of the matrix of the reduced hamiltonian

$$
\hat{H}=\boldsymbol{H} \cdot \mathbf{g} \cdot \boldsymbol{S}+D\left[S_{z}^{2}-35 / 12\right],
$$

until agreement between the calculated fine-structure spectrum and the experimental spectra was subjectively optimized. The calculated fine-structure resonance positions are indicated by the arrows in figs. 1 and 2. The calculated energy levels obtained using eq. (4) are illustrated in figs. 4-6. The positions of the fine-structure resonances illustrated in the stick spectra agree well with those observed in the X-band and Q-band spectra. The manganese $I=5 / 2$ nuclear hyperfine splitting is identical for both orientations of the field and is equal to that observed in the X-band spectrum.

The most striking difference between the ESR spectrum of $\mathrm{Mn}^{2+}$ in $\mathrm{Cd}_{2} \mathrm{P}_{2} \mathrm{~S}_{6}$ and $\mathrm{Cd}_{2} \mathrm{P}_{2} \mathrm{Se}_{6}$ is the

$$
\text { THETA }=0 \mathrm{D}=0.2863 \mathrm{CM}-1
$$

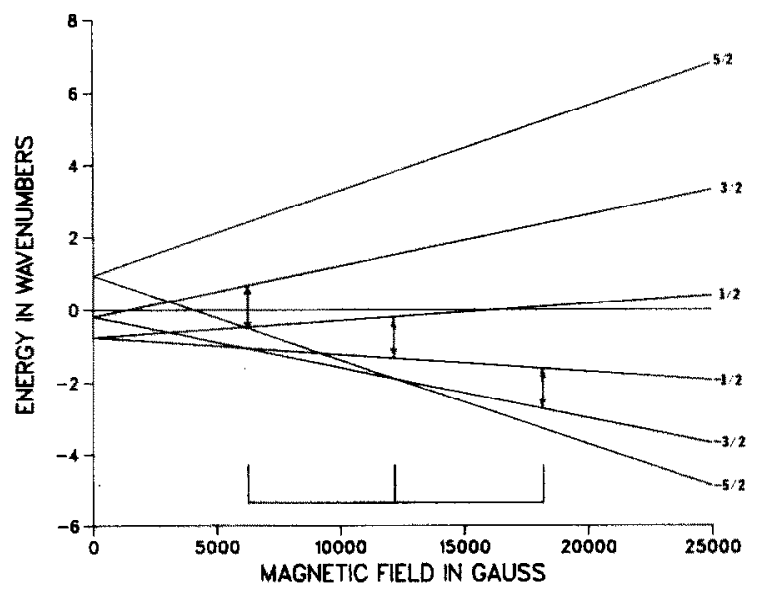

Fig. 4. Computed field dependence of the fine-structure levels of $\mathrm{Cd}_{2} \mathrm{P}_{2} \mathrm{Se}_{6}: \mathrm{Mn}$ for $H$ parallel to $c$. The positions of the predicted $Q$-band resonances are indicated in the stick spectrum. 


\section{THETA $=90 \quad D=0.2863 \mathrm{CM}-1$}

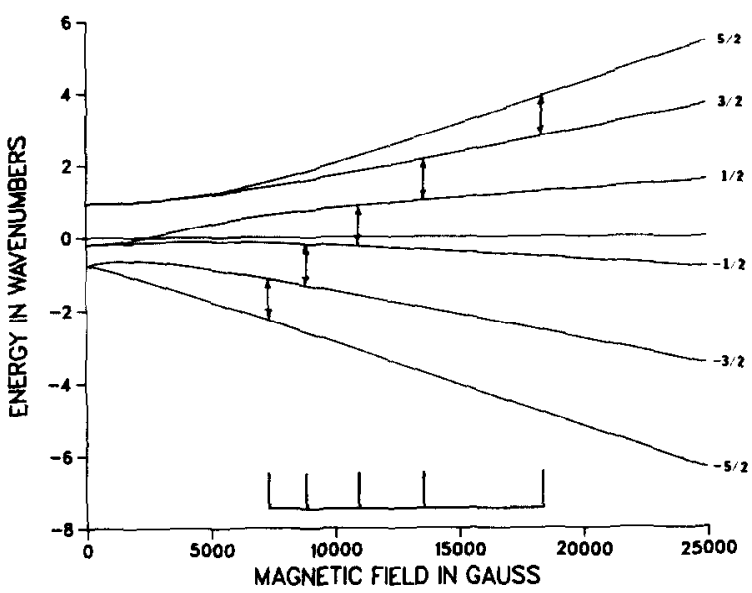

Fig. 5. Computed field dependence of the fine-structure levels of $\mathrm{Cd}_{2} \mathrm{P}_{2} \mathrm{Se}_{6}: \mathrm{Mn}$ for $H$ perpendicular to $c$. The positions of the predicted $Q$-band resonances are indicated in the stick spectrum.

nearly eight-fold increase in the axial crystal-field splitting parameter, $|D|\left(0.0365-0.2863 \mathrm{~cm}^{-1}\right)$. The changes in $g$ and the hyperfine constant $A$ are not nearly as pronounced and no further discussion of them will be presented here. Previous

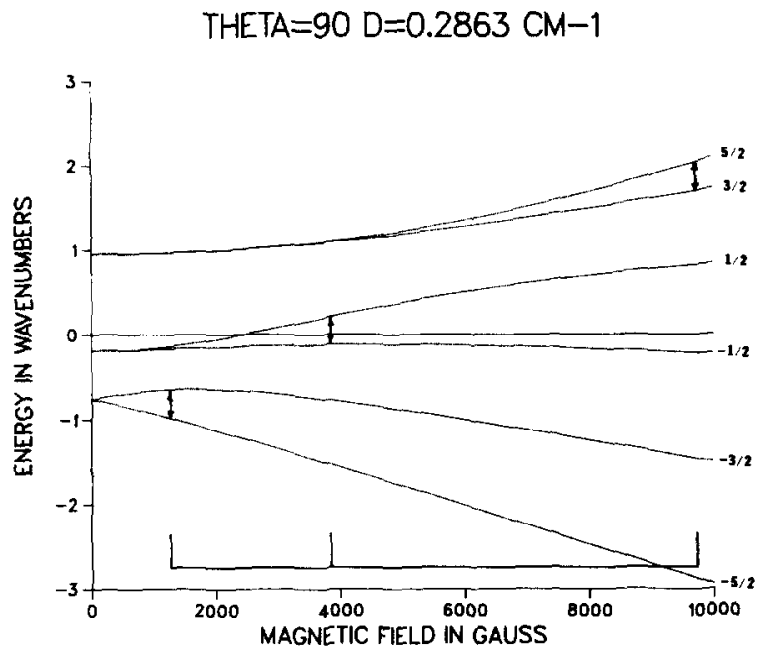

Fig. 6. Computed field dependence of the fine-structure levels of $\mathrm{Cd}_{2} \mathrm{P}_{2} \mathrm{~S}_{6}$ for $H$ perpendicular to $c$. The positions of the predicted $\mathrm{X}$-band resonances are indicated in the stick spectrum. investigations have attempted to correlate the magnitude of $|D|$ with the covalency of the $\mathrm{Mn}-\mathrm{Se}$ bond or the magnitude of the axial crystal field. These effects are discussed briefly in the following sections.

\section{2. $D$ and covalency}

The magnitude of $|D|$ is strongly dependent upon local structure. In a perfect octahedral environment $D$ is identically zero, whereas in the planar cnvironment of a porphyrin ring, $|D|$ can be as large as $20-30 \mathrm{~cm}^{-1}$ for $\mathrm{Fe}^{3+}$ [11]. The local metal environments in $\mathrm{Cd}_{2} \mathrm{P}_{2} \mathrm{~S}_{6}$ and $\mathrm{Cd}_{2} \mathrm{P}_{2} \mathrm{Se}_{6}$ are so similar that one predicts little if any change in $D$ between the two crystals due to structural differences.

Schlaak and Weiss [3] observed a similar phenomenon when investigating the ESR spectra of $\mathrm{Mn}^{2+}$ in $\mathrm{CdGa}_{2} \mathrm{~S}_{4}$ and $\mathrm{CdGa}_{2} \mathrm{Se}_{4}$. They reported a four-fold increase in $|D|$ from the sulfide to the selenide $\left(0.0225\right.$ to $\left.0.0919 \mathrm{~cm}^{-1}\right)$ and attributed this increase to the more covalent character of the $\mathrm{Mn}-\mathrm{Se}$ bond. $\mathrm{MgTe}_{2}$ yields a larger $|D|$, consistent with the expected further increase in covalency of the manganese-chalcogenide bond. Some of the larger values of $|D|$ for $\mathrm{Mn}^{2+}$ which have been reported previously, together with values from the present study, are collected in table 1. Also indicated in table 1 are the apparent major

Table 1

Large axial-field splitting parameters for $\mathrm{Mn}(\mathrm{II})$ in several lattices

\begin{tabular}{|c|c|c|c|}
\hline Host & Effect & $|D|\left(\mathrm{cm}^{-1}\right)$ & Ref. \\
\hline $\mathrm{MgTe}_{2}$ & covalency & 0.860 & [5] \\
\hline $\mathrm{SrTiO}_{3}$ & axial site & 0.544 & [4] \\
\hline $\mathrm{Ge}_{x} \mathrm{Se}_{1-x}$ & covalency & $>0.3$ & [12] \\
\hline $\mathrm{Cd}_{2} \mathrm{P}_{2} \mathrm{Se}_{6}$ & $\begin{array}{l}\text { covalency, } \\
\text { layered host }\end{array}$ & 0.286 & \\
\hline $\mathrm{NH}_{4} \mathrm{Cl}-\mathrm{MnCl}_{2}-\mathrm{H}_{2} \mathrm{O}$ & layered host & 0.150 & \\
\hline $\mathrm{ZnS} / \mathrm{ZnSe}$ & axial site & 0.1454 & [13] \\
\hline $\mathrm{CdGa}_{2} \mathrm{Se}_{4}$ & covalency & 0.0919 & [3] \\
\hline $\mathrm{Cs}_{2} \mathrm{Zn}_{3} \mathrm{~S}_{4}$ & $\begin{array}{l}\text { covalency, } \\
\text { layered host }\end{array}$ & 0.0890 & [16] \\
\hline $\mathrm{ZnSe}$ & covalency & 0.0425 & [13] \\
\hline $\mathrm{Cd}_{2} \mathrm{P}_{2} \mathrm{~S}_{6}$ & layered host & 0.0365 & \\
\hline $\mathrm{CdGa}_{2} \mathrm{~S}_{4}$ & covalency & 0.0225 & [3] \\
\hline $\mathrm{ZnS}$ & & 0.0131 & [13] \\
\hline
\end{tabular}


factors contributing to the unusual magnitude of $|D|$ as inferred from the original analysis. Where the value of $|D|$ was not determined, its magnitude has been estimated from the effective electronic $g$ value. A $g_{\text {eff }}=4.3$ at X-band is characteristic of a paramagnetic ion whose axial crystal-field splitting parameter $|D|$ is comparable in energy to the Zeeman energy term, which at X-band equals $0.30 \mathrm{~cm}^{-1}$.

\section{3. $D$ and lattice dimensionality}

Another factor that appears to influence $D$ is the dimensionality of the host lattice in which the $\mathrm{Mn}^{2+}$ is incorporated. Large values of $|D|(0.118$ and $0.181 \mathrm{~cm}^{-1}$ ) were observed by Calvo et al. [14] for $\mathrm{Mn}^{2+}$ in $\mathrm{Mg}_{2} \mathrm{P}_{2} \mathrm{O}_{7}$. The structure of this material has a substantial two-dimensional character. The $\mathrm{Mn}^{2+}$ replaces the $\mathrm{Mg}^{2+}$ and is coordinated by six oxygens. Four of the six oxygens are coplanar and form extended two-dimensional sheets perpendicular to the principle magnetic axis. Although the $\mathrm{Mn}-\mathrm{O}$ bonds are less covalent than either Mn-S or Mn-Se bonds, the observed $|D|$ value is quite large, $0.1814 \mathrm{~cm}^{-1}$.

Seed [15] reported a large $|D|$ for $\left(0.15 \mathrm{~cm}^{-1}\right)$ $\mathrm{Mn}^{2+}$ in the ternary system $\mathrm{NH}_{4} \mathrm{Cl}-\mathrm{MnCl}_{2}-\mathrm{H}_{2} \mathrm{O}$, which the author reports to be characterized by layered growth. This is another example of an $\mathrm{Mn}-\mathrm{O}$ bond where covalency is not expected to contribute substantially to a large value of $|D|$. The influence of a lamellar lattice structure is also mentioned by Heming and Lehmann [16] in a study of $\mathrm{Mn}^{2+}$ in $\mathrm{Cs}_{2} \mathrm{Zn}_{3} \mathrm{~S}_{4}$. They conclude that the layered structure increased the covalency of the $\mathrm{Mn}-\mathrm{S}$ bond in $\mathrm{Cs}_{2} \mathrm{Zn}_{3} \mathrm{~S}_{4}$ relative to the same bond in three-dimensional binary sulfides and $\mathrm{CdGa}_{2} \mathrm{~S}_{4}$. While it would be difficult to show that the $\mathrm{Mn}-\mathrm{S}$ bond is substantially more covalent, it is clear that the macroscopic two-dimensional structure of the host is important in determining the magnitude of $|D|$.

\subsection{The superposition model}

Newman and Urban [17] proposed a model for computation of the crystal-field splitting in which the parameter $D$ is determined entirely by the first coordination sphere of the paramagnetic ion. The superposition model (SPM) has been applied successfully in several instances. For example, Heming et al. [18] have applied the model to a series of $\mathrm{Mn}^{2+}$ coordination compounds with $\mathrm{F}^{-}, \mathrm{Cl}^{-}$and $\mathrm{O}^{2-}$ as ligands.

According to the SPM, the axial splitting term $D$ is obtained from:

$D=\frac{1}{2} \sum_{i}\left(3 \cos ^{2} \phi_{i}-1\right)\left(R_{0} / R_{i}\right)^{7} b_{2}$.

The crystal structure data are used to determine $\phi_{i}$ and $R_{i}, R_{0}$ is a value of $R_{i}$ for which $b_{2}$ is known. The parameter $b_{2}$ is the intrinsic zero-field splitting which is calculated from the ESR spectra. The idea behind the theory is that $b_{2}$ for a given ligand will be essentially the same for all occurrences of that ligand. It should be noted that in all cases where the SPM is applicable, the splitting parameter $D$ is much less than the Zeeman term. One reason for this is that contributions from covalency are superposable as long as chargetransfer integrals remain small quantities [19].

Lehmann and co-workers [18-20] have demonstrated the validity of the SPM in a series of $\mathrm{Mn}^{2+}$ coordination compounds. They calculated the spin-spin and spin-orbit contributions to the intrinsic splitting parameter $b_{2}$, and compared the individual overlap and covalency contributions to $b_{2}$ for $\mathrm{Mn}^{2+}-\mathrm{F}^{-}$and $\mathrm{Mn}^{2+}-\mathrm{Cl}^{-}$[19]. Additionally, they have examined the effects of coordination number, bond covalency and site distortion upon the crystal-field splitting $[19,20]$.

Unfortunately, the superposition model provides little help in either predicting the ESR spectrum of $\mathrm{Mn}^{2+}$ in $\mathrm{Cd}_{2} \mathrm{P}_{2} \mathrm{Se}_{6}$ or in interpreting it. The problem with the model is the intrinsic parameter $b_{2}$, which carries all the effects of covalency, charge transfer, configuration interaction, etc. There are currently no reported values of $b_{2}$ for $\mathrm{Se}^{2-}$ with which to evaluate the SPM for the $\mathrm{Cd}_{2} \mathrm{P}_{2} \mathrm{Se}_{6}: \mathrm{Mn}$ system. The only value of $b_{2}$ reported to date for $\mathrm{Mn}^{2+}-\mathrm{S}^{2-}$ in a layered compound is $0.14 \mathrm{~cm}^{-1}$ [20]. In this case, the manganese is coordinated to four sulfurs, while in $\mathrm{Cd}_{2} \mathrm{P}_{2} \mathrm{~S}_{6}$ it is bound to six. The value of $b_{2}$ for $\mathrm{Mn}^{2+}$ in $\mathrm{Cd}_{2} \mathrm{P}_{2} \mathrm{~S}_{6}$ is $0.06 \mathrm{~cm}^{-1}$, and the reduction in $b_{2}$ by $\approx 50 \%$ in going from four-coordination 
to six-coordination is consistent with the results of Lehmann and co-workers [21]. The value of $b_{2}$ in $\mathrm{Cd}_{2} \mathrm{P}_{2} \mathrm{Se}_{6}: \mathrm{Mn}^{2+}$ is $0.45 \mathrm{~cm}^{-1}$, and no provision of the model leads to this. The superposition model appears to be most applicable when the environment of the paramagnetic ion under study is similar to a previously studied environment, both structurally and chemically.

\subsection{Theoretical estimates of $D$}

A relationship between bond covalency and $D$ has been suggested by several authors, however, an unambiguous theoretical model for the effect has not been established. It is possible that covalency directly affects mixing of ground state and excited state wavefunctions. Pryce [22] showed that a small contribution to the zero-field splitting could be obtained through admixture of excited state $S=5 / 2$ configurations (e.g., $3 d^{4} 4 s^{1}$ ) by use of the spin-spin coupling. A similar interaction could occur with excited charge-transfer states having $S=5 / 2$. Watanabe [23] considered admixture of excited states of the $\mathrm{d}^{5}$ ground state configuration with $S<5 / 2$ through the spin-orbit coupling interaction. Kondo [24] computed the covalent contributions to the axial-field splitting and obtained reasonable agreement with relatively small values of the sigma bonding electron transfer coefficient. These results suggested that the overlap contribution to the axial-field splitting was dominant. The "Blume-Orbach" mechanism [25] accounts for the magnitude of $|D|$ in $\mathrm{ZnF}_{2}$ and $\mathrm{MnF}_{2}$. The mechanism involves first-order matrix elements of the axial and rhombic fields between excited quartet states which have been admixed into the ground state by spin-orbit coupling.

\section{Conclusion}

In the $\mathrm{Cd}_{2} \mathrm{P}_{2} \mathrm{Se}_{6}$ lattice, both the highly two-dimensional nature of the layered lattice and the covalency of the $\mathrm{Mn}$-Se bond increase the magnitude of $|D|$. Because the electronegativity of the chalcogenides increases in the order $\mathrm{S}>\mathrm{Se}>\mathrm{Te}$, the $\mathrm{Mn}-\mathrm{S}$ bond in $\mathrm{CdGa}_{2} \mathrm{~S}_{4}$ and $\mathrm{Cd}_{2} \mathrm{P}_{2} \mathrm{~S}_{6}$ are less covalent than the $\mathrm{Mn}-\mathrm{Se}$ bond in $\mathrm{CdGa}_{2} \mathrm{Se}_{4}$ and $\mathrm{Cd}_{2} \mathrm{P}_{2} \mathrm{Se}_{6}$. This is reflected in the large increase in the zero-field splitting parameter in the selenide versus the sulfide.

\section{Acknowledgement}

Partial funding for this research was provided by the Donors of the Petroleum Research Fund, administered by the American Chemical Society, and by the National Science Foundation under grant \# CHE-8200857. EL received support from a Sir Charles Clore Post Doctoral Fellowship at the Weizmann Institute of Science and DC was partially supported by a Sokol Fellowship at the University of Michigan.

\section{References}

[1] R.R. Sharma, T.P. Das and R. Orbach, Phys. Rev. 171 (1968) 378; 155 (1967) 338; 149 (1966) 257.

[2] W.J. Nicholson and G. Burns, Phys. Rev. 129 (1963) 2490.

[3] M. Schlaak and A. Weiss, Z. Naturforsch. 27a (1972) 1624.

[4] R.A. Serway, W. Berlinger, K.A. Mullker and R.W. Collins, Phys. Rev. B16 (1977) 4761.

[5] O. Okada and T. Miyadai, Japan. J. Phys. 17 (1978) 231.

[6] B. Bleaney and R.S. Trenam, Proc. Roy. Soc. A223 (1954) 1;

S. Geschwind, Phys. Rev. 121 (1961) 363.

[7] E. Meirovitch and R. Poupko, J. Phys. Chem. 82 (1978) 1920.

[8] F. I.ifshitz, Ph.D. Dissertation, University of Michigan, USA (1983).

[9] E. Lifshitz and A.H. Francis, J. Phys. Chem. 86 (1982) 4714.

[10] E. Lifshitz, A.E. Gentry and A.H. Francis, J. Phys. Chem. 88 (1984) 3038.

[11] J.E. Bennett, J.F. Gibson and D.J.E. Ingram, Proc. Roy. Soc. A240 (1957) 67.

[12] R. Durnỳ, J. Non-Cryst. Solids 41 (1980) 273.

[13] J. Schneider, B. Dischler and A. Räuber, J. Phys. Chem. Solids 29 (1968) 451.

[14] C. Calvo, J.S. Leung and W.R. Datars, J. Chem. Phys. 46 (1967) 796.

[15] T.J. Seed, J. Chem. Phys. 41 (1964) 1486.

[16] M. Heming and G. Lehmann, Z. Naturforsch. 38a (1982) 149.

[17] D.J. Newman and W. Urban, Advan. Phys. 24 (1975) 793.

[18] G. Lehmann, Phys. Stat. Sol. 99b (1980) 623.

[19] M. Heming, S. Remme and G. Lehmann, Ber. Bunsenges. Physik. Chem. 88 (1984) 946.

[20] M. Heming, G. Lehmann, H. Mosebach and E. Siegel, Solid State Commun. 44 (1982) 543.

[21] M. Heming and G. Lehman, Chem. Phys. Letters 80 (1981) 235.

[22] M.H.L. Pryce, Phys. Rev. 80 (1950) 1107.

[23] H. Watanabe, Progr. Theoret. Phys. (Kyoto) 18 (1957) 405.

[24] J. Kondo, Progr. Theoret. Phys. (Kyoto) 23 (1960) 106; 28 (1962) 1026.

[25] M. Blume and R. Orbach, Phys. Rev. 127 (1962) 1587. 